\title{
3D/2D Image Registration: The Impact of X-Ray Views and Their Number
}

\author{
Dejan Tomaževič, Boštjan Likar, and Franjo Pernuš \\ University of Ljubljana, Faculty of Electrical Engineering \\ Tržaška 25, 1000 Ljubljana, Slovenia \\ Tel.: +38614768 248, Fax: +38614768279 \\ \{dejan.tomazevic, bostjan.likar, \\ franjo.pernus\}@fe.uni-lj.si
}

\begin{abstract}
An important part of image-guided radiation therapy or surgery is registration of a three-dimensional (3D) preoperative image to two-dimensional (2D) images of the patient. It is expected that the accuracy and robustness of a 3D/2D image registration method do not depend solely on the registration method itself but also on the number and projections (views) of intraoperative images. In this study, we systematically investigate these factors by using registered image data, comprising of $\mathrm{CT}$ and X-ray images of a cadaveric lumbar spine phantom and the recently proposed $3 \mathrm{D} / 2 \mathrm{D}$ registration method [1], [2]. The results indicate that the proportion of successful registrations (robustness) significantly increases when more X-ray images are used for registration.
\end{abstract}

Keywords: 3D/2D image registration, computed tomography, image-guided surgery, X-ray images.

\section{Introduction}

In radiation therapy and surgery, there is a constant demand to render the therapeutic procedures less and less invasive and to improve the accuracy with which a given procedure can be performed compared to conventional methods. Image guidance is the emerging technology that has the potential to decrease the invasiveness and increase the accuracy of procedures [3].The crucial part of image-guided systems that enable intra-therapy patient setup or provide intraoperative navigation guidance is registration of a patient in the treatment room to preoperative patient images or to models obtained from these images. A variety of rigid registration techniques have been proposed in the past that may be classified according to the data they use to compute the registration transformation [2], [4]. Geometry-based methods use points [5] or surfaces [6], [7], while intensity-based methods use contours of anatomical structures obtained by preoperative image segmentation [8] or image voxel intensity or gradient information [2], [4], [9]. Most of these techniques implement rigid registration of a 3D image to $2 \mathrm{D}$ images, while another set of methods exists, where a $3 \mathrm{D}$ anatomy model is non-rigidly registered to intraoperative $2 \mathrm{D} \mathrm{X}$-ray images $[10$, 
11]. The methods that register preoperative $3 \mathrm{D}$ images/models to one or more intraoperative $2 \mathrm{D}$ images are commonly referred to as $3 \mathrm{D} / 2 \mathrm{D}$ or $2 \mathrm{D} / 3 \mathrm{D}$ registration methods. The accuracy and robustness of a 3D/2D registration method based on intraoperative X-ray images don't depend solely on the registration method but also on the specific anatomy, projections (views) and the number of X-ray images used for registration. To the best of our knowledge, we are not aware of any systematic study that would report on the impact of X-ray views and their number on the performance of a $3 \mathrm{D} / 2 \mathrm{D}$ registration method. In the present study, we have therefore used image data, comprising of a CT and 18 X-ray images of a cadaveric lumbar spine phantom [1] and the validation protocol and validation metrics [2] to study the impact of the number of intraoperative X-ray images and selected projections (views) on the capture range and registration accuracy and robustness of our recently proposed $3 \mathrm{D} / 2 \mathrm{D}$ registration method [2].

\section{3D/2D Registration Method}

Features that we use for rigid 3D/2D registration are normals $\mathbf{v}_{\mathrm{A}}$ to surfaces of bony structures found in preoperative CT volumes and back-projected intensity gradients $\mathbf{v}_{\mathrm{B}}$ of intraoperative X-ray images (Fig. 1) [2]. Let $\mathbf{r}_{\mathrm{i}}{ }^{\mathrm{v}}$, a point on the surface of a 3D structure, be defined in the coordinate system $S_{\mathrm{v}}$ of a CT volume (Fig. 1). The position $\mathbf{r}_{\mathrm{i}}$ of the same point in reference (patient) coordinate system $\boldsymbol{S}_{\text {ref }}$ is given by rigid transformation $\mathbf{T}$ defined by six parameters $\mathbf{q}=\left(t_{x}, t_{y}, t_{z}, \omega_{x}, \omega_{y}, \omega_{z}\right)^{\mathrm{T}}$

$$
\mathbf{r}_{\mathrm{i}}^{S_{\text {ref }}}=\mathbf{r}_{\mathrm{i}}=\mathbf{T}\left(\mathbf{r}_{\mathrm{i}}^{S_{\mathrm{v}}}\right)=\mathbf{R} \mathbf{r}_{\mathrm{i}}^{S_{\mathrm{v}}}+\mathbf{t}
$$

where $\mathbf{R}$ and $\mathbf{t}$ describe, respectively, the rotation and translation of coordinate system $S_{\mathrm{v}}$ with respect to $\boldsymbol{S}_{\mathrm{ref}}$. Let $\mathbf{r}_{\mathrm{s}}$ be the position of the X-ray source in the reference coordinate system $S_{\text {ref. }}$. For a given position of a 3D image, defined by vector $\mathbf{q}$, the line which connects $\mathbf{r}_{\mathrm{s}}$ and $\mathbf{r}_{\mathrm{i}}$ and has direction defined by unit vector $\mathbf{e}_{\mathrm{i}}$, intersects the X-ray image plane $\mathrm{U}$ at point $\mathbf{p}_{\mathrm{i}}=\mathbf{p}\left(\mathbf{r}_{\mathrm{i}}\right)$ (Fig. 1).
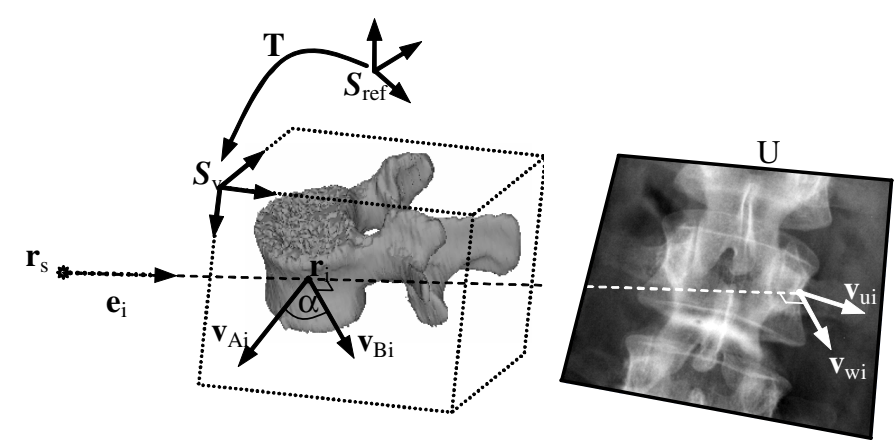

Fig. 1. 3D/2D registration geometrical setup 
Rigid registration of a $3 \mathrm{D}$ preoperative image to $M$ intraoperative $2 \mathrm{D}$ images is concerned with finding the set of parameters $\mathbf{q}$ that optimizes the criterion function $C F$

$$
C F=\sum_{\mathrm{j}=1}^{M} \frac{\sum_{\mathrm{i}=1}^{N}\left|\mathbf{v}_{\mathrm{Ai}}^{\mathrm{j}}\right| \cdot\left|\mathbf{v}_{\mathrm{Bi}}^{\mathrm{j}}\right| \cdot f(\alpha)}{\sum_{\mathrm{i}=1}^{\mathrm{N}}\left|\mathbf{v}_{\mathrm{Ai}}^{\mathrm{j}}\right| \cdot \sum_{\mathrm{i}=1}^{\mathrm{N}}\left|\mathbf{v}_{\mathrm{Bi}}^{\mathrm{j}}\right|}
$$

where $N$ is the number of surface points $\mathbf{r}_{\mathrm{i}}{ }^{S_{\mathrm{v}}}, \mathbf{v}_{\mathrm{Ai}}$ is the vector normal to the surface at point $\mathbf{r}_{\mathrm{i}}^{S \mathrm{v}}, \mathbf{v}_{\mathrm{Bi}}$ is the X-ray image gradient $\mathbf{v}_{\mathrm{Ui}}$ back-projected to the $\mathbf{r}_{\mathrm{i}}{ }^{S \mathrm{v}}$, and $f(\alpha)$ is the weighting function depending on angle $\alpha$ between $\mathbf{v}_{\mathrm{Ai}}$ and $\mathbf{v}_{\mathrm{Bi}}$. The gradient $\mathbf{v}_{\mathrm{Bi}}$ is obtained by back-projecting the gradient $\mathbf{v}_{\mathrm{Ui}}\left(\mathbf{p}_{\mathrm{i}}\right), \mathbf{v}_{\mathrm{Ui}}\left(\mathbf{p}_{\mathrm{i}}\right)=\operatorname{grad}_{\mathrm{U}} I\left(\mathbf{p}_{\mathrm{i}}\right)$, of X-ray image intensity $I\left(\mathbf{p}_{\mathrm{i}}\right)$

$$
\mathbf{v}_{\mathrm{Bi}}\left(\mathbf{r}_{\mathrm{i}}\right)=\frac{\left|\mathbf{p}_{\mathrm{i}}-\mathbf{r}_{\mathrm{s}}\right|}{\left|\mathbf{r}_{\mathrm{i}}-\mathbf{r}_{\mathrm{s}}\right|} \cdot \mathbf{v}_{\mathrm{w}}(\mathbf{p})=\frac{\left|\mathbf{p}_{\mathrm{i}}-\mathbf{r}_{\mathrm{s}}\right|}{\left|\mathbf{r}_{\mathrm{i}}-\mathbf{r}_{\mathrm{s}}\right|} \cdot \frac{\left(\mathbf{n} \times \mathbf{v}_{\mathrm{Ui}}\left(\mathbf{p}_{\mathrm{i}}\right)\right) \times \mathbf{e}_{\mathrm{i}}}{\mathbf{n} \cdot \mathbf{e}_{\mathrm{i}}}
$$

where $\left|\mathbf{r}_{\mathbf{i}}-\mathbf{r}_{\mathrm{s}}\right|$ and $\left|\mathbf{p}_{\mathbf{i}}-\mathbf{r}_{\mathrm{s}}\right|$ are the distances from X-ray source $\mathbf{r}_{\mathrm{s}}$ to points $\mathbf{r}_{\mathbf{i}}$ and $\mathbf{p}_{\mathbf{i}}$, respectively, $\quad \mathbf{v}_{\mathrm{w}}\left(\mathbf{p}_{\mathbf{i}}\right), \quad \mathbf{v}_{\mathrm{w}}\left(\mathbf{p}_{\mathrm{i}}\right)=\operatorname{grad}_{\mathrm{w}} I\left(\mathbf{p}_{\mathbf{i}}\right)$, is the intensity gradient in the plane perpendicular to projection beam defined by $\mathbf{e}_{\mathbf{i}}$, and $\mathbf{n}$ is the unit normal to projection plane $U$.
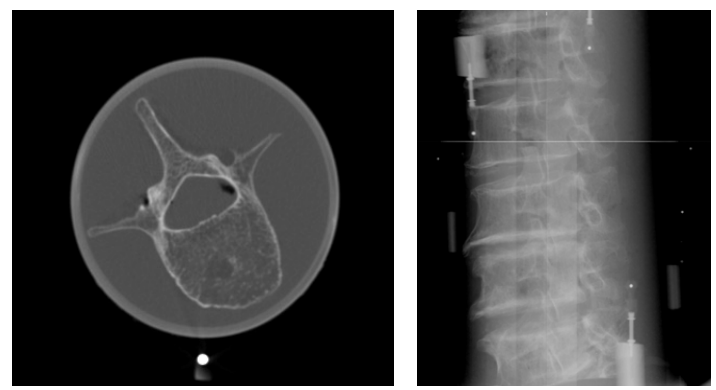

Fig. 2. Axial slice from a CT (left) and one of the 18 acquired X-ray images (right) of the spine phantom

\section{Images and "Gold Standard" Registrations}

Images used in the experiment were part of our 3D/2D registration "gold standard" lumbar spine phantom data set, which has been made publicly available [1]. A lumbar spine phantom had been constructed by placing a cadaveric lumbar spine, comprising vertebra L1-L5 with intervertebral disks and several millimeters of soft tissue, into a plastic tube filled with water. Six fiducial markers were rigidly attached to the surface of the tube. The CT image was obtained using a General Electric HiSpeed CT/i scanner. Axial slices were taken with intra-slice resolution of $0.27 \times 0.27 \mathrm{~mm}$ and 1 $\mathrm{mm}$ inter-slice distance. X-ray images were obtained by PIXIUM 4600 (Trixell) 
digital X-ray detector with a 429 x $429 \mathrm{~mm}$ large active surface, 0.143 x $0.143 \mathrm{~mm}$ pixel size, and 14 bits of dynamic range. The X-ray source and detector-plane were fixed during image acquisition while the spine phantom was rotated on a turntable to simulate a setup with $\mathrm{C}$-arm. By rotating $\left(\mathrm{step}=20^{\circ}\right)$ the spine phantom around its long axis, $18 \mathrm{X}$-ray images were acquired. "Gold standard" registrations of CT to Xray images were obtained by rigid registration of $\mathrm{CT}$ marker points to 3D marker points reconstructed from X-ray images, respectively. As such, the obtained "gold standard" registration defines the relative position of each of the X-ray images to CT volume and, consequently, relative positions between X-ray images. After registration, cubic sub-images of single vertebrae without markers, were defined manually in CT images. Each sub-volume was blurred using a Gaussian filter $(\sigma=0.5$ $\mathrm{mm}$ ) and isotropically re-sampled to the resolution of $1 \mathrm{~mm}$. The Canny edge detector and a threshold were applied to automatically extract locations of points on surfaces of bony structures and to estimate surface normal directions at these points. The X-ray images were blurred with a Gaussian filter $(\sigma=0.5 \mathrm{~mm})$ and the Roberts edge detector was applied to calculate intensity $\operatorname{gradients}_{\operatorname{grad}} I(\mathbf{p})$.

\section{Experiments}

To test the impact of X-ray views and their number on the accuracy and robustness of the $3 \mathrm{D} / 2 \mathrm{D}$ registration method, registrations were performed from a wide range of starting positions and orientations around the "gold standard" registration position using different projections and different numbers of X-ray images. Before registrations, the 6-dimensional parametrical space was normalized, so that a rotation of the volume containing a single vertebra of size $80 \mathrm{~mm}$ around its center for 0.1 radians $\left(5.7^{\circ}\right)$ was equivalent to mean translation of volume points for $2 \mathrm{~mm}$. In this way, Euclidean metrics could be used to calculate the displacement (in parametrical space) of a starting position from the "gold standard" position [2]. Values of parameters defining a starting point were chosen randomly within $18 \mathrm{~mm}\left(51.6^{\circ}\right)$ around the "gold standard" position. Optimization of transformation parameters $\mathbf{q}=\left(t_{x}\right.$, $\left.t_{y}, t_{z}, \omega_{x}, \omega_{y}, \omega_{z}\right)^{\mathrm{T}}$ was performed by Powel's method. To measure the registration error before and after registration, target registration error (TRE) [12] was calculated for eight target points (four on each pedicle) as the distance between target points in registered and "gold standard" position. Sixteen X-ray image sets, each containing 18 image subsets were formed (Table 1). The first set contained the 18 single images. Subsets in the next eight sets were comprised of image pairs taken at views, which differed by $20^{\circ}, 40^{\circ}, \ldots, 160^{\circ}$, respectively. Subsets in the next four sets contained three images. The angles between the three images in a subset were $20^{\circ}, 40^{\circ}, 60^{\circ}$, or $80^{\circ}$. In the next two subsets of set 4 there were four images, the angles between them were $20^{\circ}$ and $40^{\circ}$. In the 18 subsets of the last set there were eight images taken at views $20^{\circ}$ apart. The $5 \mathrm{CT}$ sub-volumes, each containing one of the vertebra L1-L5, were registered from 50 initial positions to each of the 18 subsets of X-ray images from all 16 image sets, which yielded $4500(5 \times 50 \times 18)$ registrations for each set. 
Table 1. The number and angle between two consecutive views in 16 sets of X-ray images registered to $\mathrm{CT}$ images

\begin{tabular}{|ccc|c|c|c|c|}
\hline \multicolumn{7}{|c|}{ X-ray images } \\
\hline \multicolumn{3}{|c|}{ Set } & & \multicolumn{4}{c|}{ Subsets } \\
\hline No. & $\mathbf{M}$ & $\boldsymbol{\Delta}$ & $\mathbf{1}$ & $\mathbf{2}$ & $\ldots$ & $\mathbf{1 8}$ \\
\hline $\mathbf{1}$ & $\mathbf{1}$ & & $\left(0^{\circ}\right)$ & $\left(20^{\circ}\right)$ & $\ldots$ & $\left(340^{\circ}\right)$ \\
\hline $\mathbf{2}$ & $\mathbf{2}$ & $\mathbf{2 0}^{\circ}$ & $\left(0^{\circ}, 20^{\circ}\right)$ & $\left(20^{\circ}, 40^{\circ}\right)$ & $\ldots$ & $\left(340^{\circ}, 0^{\circ}\right)$ \\
$\mathbf{3}$ & & $\mathbf{4 0}^{\circ}$ & $\left(0^{\circ}, 40^{\circ}\right)$ & $\left(20^{\circ}, 60^{\circ}\right)$ & $\ldots$ & $\left(340^{\circ}, 20^{\circ}\right)$ \\
$\mathbf{4}$ & & $\mathbf{6 0}^{\circ}$ & $\left(0^{\circ}, 60^{\circ}\right)$ & $\left(20^{\circ}, 80^{\circ}\right)$ & $\ldots$ & $\left(340^{\circ}, 40^{\circ}\right)$ \\
$\mathbf{5}$ & & $\mathbf{8 0}^{\circ}$ & $\left(0^{\circ}, 80^{\circ}\right)$ & $\left(20^{\circ}, 100^{\circ}\right)$ & $\ldots$ & $\left(340^{\circ}, 60^{\circ}\right)$ \\
$\mathbf{6}$ & & $\mathbf{1 0 0}^{\circ}$ & $\left(0^{\circ}, 100^{\circ}\right)$ & $\left(20^{\circ}, 120^{\circ}\right)$ & $\ldots$ & $\left(340^{\circ}, 80^{\circ}\right)$ \\
$\mathbf{7}$ & & $\mathbf{1 2 0}^{\circ}$ & $\left(0^{\circ}, 120^{\circ}\right)$ & $\left(20^{\circ}, 140^{\circ}\right)$ & $\ldots$ & $\left(340^{\circ}, 100^{\circ}\right)$ \\
$\mathbf{8}$ & & $\mathbf{1 4 0}^{\circ}$ & $\left(0^{\circ}, 140^{\circ}\right)$ & $\left(20^{\circ}, 160^{\circ}\right)$ & $\ldots$ & $\left(340^{\circ}, 120^{\circ}\right)$ \\
$\mathbf{9}$ & & $\mathbf{1 6 0}^{\circ}$ & $\left(0^{\circ}, 160^{\circ}\right)$ & $\left(20^{\circ}, 180^{\circ}\right)$ & $\ldots$ & $\left(340^{\circ}, 140^{\circ}\right)$ \\
\hline $\mathbf{1 0}$ & $\mathbf{3}$ & $\mathbf{2 0}^{\circ}$ & $\left(0^{\circ}, 20^{\circ}, 40^{\circ}\right)$ & $\left(20^{\circ}, 40^{\circ}, 60^{\circ}\right)$ & $\ldots$ & $\left(340^{\circ}, 0^{\circ}, 20^{\circ}\right)$ \\
$\mathbf{1 1}$ & & $\mathbf{4 0}^{\circ}$ & $\left(0^{\circ}, 40^{\circ}, 80^{\circ}\right)$ & $\left(20^{\circ}, 60^{\circ}, 100^{\circ}\right)$ & $\ldots$ & $\left(340^{\circ}, 20^{\circ}, 60^{\circ}\right)$ \\
$\mathbf{1 2}$ & & $\mathbf{6 0}^{\circ}$ & $\left(0^{\circ}, 60^{\circ}, 120^{\circ}\right)$ & $\left(20^{\circ}, 80^{\circ}, 140^{\circ}\right)$ & $\ldots$ & $\left(340^{\circ}, 40^{\circ}, 100^{\circ}\right)$ \\
$\mathbf{1 3}$ & & $\mathbf{8 0}^{\circ}$ & $\left(0^{\circ}, 80^{\circ}, 160^{\circ}\right)$ & $\left(20^{\circ}, 100^{\circ}, 180^{\circ}\right)$ & $\ldots$ & $\left(340^{\circ}, 60^{\circ}, 140^{\circ}\right)$ \\
\hline $\mathbf{1 4}$ & $\mathbf{4}$ & $\mathbf{2 0}^{\circ}$ & $\left(0^{\circ}, 20^{\circ}, 40^{\circ}, 60^{\circ}\right)$ & $\left(20^{\circ}, 40^{\circ}, 60^{\circ}, 80^{\circ}\right)$ & $\ldots$ & $\left(340^{\circ}, 0^{\circ}, 20^{\circ}, 40^{\circ}\right)$ \\
$\mathbf{1 5}$ & & $\mathbf{4 0}^{\circ}$ & $\left(0^{\circ}, 40^{\circ}, 80^{\circ}, 120^{\circ}\right)$ & $\left(20^{\circ}, 60^{\circ}, 100^{\circ}, 140^{\circ}\right)$ & $\ldots$ & $\left(340^{\circ}, 20^{\circ}, 60^{\circ}, 100^{\circ}\right)$ \\
\hline $\mathbf{1 6}$ & $\mathbf{8}$ & $\mathbf{2 0}^{\circ}$ & $\left(0^{\circ}, 20^{\circ}, \ldots, 140^{\circ}\right)$ & $\left(20^{\circ}, 40^{\circ}, \ldots, 160^{\circ}\right)$ & $\ldots$ & $\left(340^{\circ}, 0^{\circ}, \ldots, 120^{\circ}\right)$ \\
\hline
\end{tabular}

$M$ - number of images in a subset, $\Delta$ - angle between consecutive images in a subset .

\section{Results}

Table 2 shows means and standard deviations of TREs before and after successful (TRE of all 8 targets smaller than $2 \mathrm{~mm}$ ) registration and the proportion of successful registrations for three intervals of displacements. The results show that the registration errors fell with the higher number of images utilized for registration. The mean TRE achieved with one image was $0.9 \mathrm{~mm}$, with 2 images between 0.32 and 0.41 , and with 8 images $0.3 \mathrm{~mm}$. The accuracy of registrations did not significantly depend on the projections. For instance, when registering image pairs to CT images, the best results were achieved with X-ray images being 80 or 100 degrees apart. However, the values for 80 and 100 degrees $(0.32 \mathrm{~mm})$ were not significantly smaller than the values for other angles between image pairs. The percentage of successful registrations increased with the higher number of images used for registration. It increased from $96 \%$ for two images to $99.5 \%$ for eight images if the initial displacements were between 0 and $6 \mathrm{~mm}$. For the initial displacements between 6 and $12 \mathrm{~mm}$ the increase was much more dramatic. The proportion of successful registrations increased from $30 \%$ (one image), to $59 \%$ (two images), to $66 \%$ (three images), to $79 \%$ (four images) and, finally, to $80 \%$ for eight images. The same trend could be observed for displacements between 12 and $18 \mathrm{~mm}$ although, even with eight images the proportion of successful registrations was smaller than $40 \%$. 
Table 2. Registration errors and proportion of successful registrations for CT to X-ray registration using different $\mathrm{X}$-ray projections and number of projections

\begin{tabular}{|c|c|c|c|c|c|c|c|}
\hline \multicolumn{3}{|c|}{ Sets } & \multirow{2}{*}{$\begin{array}{c}\begin{array}{c}\text { Before } \\
\text { registration }\end{array} \\
\text { TRE }[\mathrm{mm}] \\
\text { Mean }(\mathrm{Std})\end{array}$} & \multirow{2}{*}{$\begin{array}{c}\begin{array}{c}\text { After } \\
\text { registration }\end{array} \\
\text { TRE }[\mathrm{mm}] \\
\text { Mean }(\mathrm{Std})\end{array}$} & \multicolumn{3}{|c|}{ Successful registrations $(\%)$} \\
\hline No. & $\mathbf{M}$ & $\Delta$ & & & $\begin{array}{l}0 \div 6 \mathrm{~mm} \\
0 \div 17.2^{\circ}\end{array}$ & $\begin{array}{c}6 \div 12 \mathrm{~mm} \\
17.2 \div 34.4^{\circ}\end{array}$ & $\begin{array}{r}12 \div 18 \mathrm{~mm} \\
34.4 \div 51.7^{\circ}\end{array}$ \\
\hline 1 & 1 & & $3.3(2.9)$ & $0.90(0.49)$ & $61.8 \%$ & $30.6 \%$ & $13.5 \%$ \\
\hline 2 & 2 & $20^{\circ}$ & $5.2(3.7)$ & $0.41(0.18)$ & $96.4 \%$ & $56.4 \%$ & $13.7 \%$ \\
\hline 3 & & $40^{\circ}$ & $5.1(3.6)$ & $0.34(0.13)$ & $95.6 \%$ & $55.4 \%$ & $13.1 \%$ \\
\hline 4 & & $60^{\circ}$ & $5.0(3.5)$ & $0.33(0.11)$ & $95.2 \%$ & $52.7 \%$ & $11.3 \%$ \\
\hline 5 & & $80^{\circ}$ & $4.9(3.5)$ & $0.32(0.11)$ & $94.7 \%$ & $52.0 \%$ & $10.5 \%$ \\
\hline 6 & & $100^{\circ}$ & $4.9(3.5)$ & $0.32(0.12)$ & $94.2 \%$ & $48.3 \%$ & $11.2 \%$ \\
\hline 7 & & $120^{\circ}$ & $5.0(3.6)$ & $0.34(0.11)$ & $93.8 \%$ & $52.1 \%$ & $12.3 \%$ \\
\hline 8 & & $140^{\circ}$ & $5.1(3.6)$ & $0.34(0.12)$ & $95.8 \%$ & $55.0 \%$ & $13.6 \%$ \\
\hline 9 & & $160^{\circ}$ & $5.3(3.7)$ & $0.40(0.17)$ & $95.3 \%$ & $59.0 \%$ & $15.1 \%$ \\
\hline 10 & 3 & $20^{\circ}$ & $5.5(3.8)$ & $0.34(0.13)$ & $98.4 \%$ & $63.6 \%$ & $18.6 \%$ \\
\hline 11 & & $40^{\circ}$ & $5.4(3.7)$ & $0.31(0.11)$ & $98.6 \%$ & $64.5 \%$ & $16.4 \%$ \\
\hline 12 & & $60^{\circ}$ & $5.5(3.8)$ & $0.31(0.11)$ & $97.9 \%$ & $66.0 \%$ & $19.4 \%$ \\
\hline 13 & & $80^{\circ}$ & $5.4(3.7)$ & $0.31(0.12)$ & $97.7 \%$ & $64.4 \%$ & $18.2 \%$ \\
\hline 14 & 4 & $20^{\circ}$ & $5.8(3.9)$ & $0.32(0.11)$ & $98.9 \%$ & $69.0 \%$ & $23.8 \%$ \\
\hline 15 & & $40^{\circ}$ & $5.7(3.9)$ & $0.30(0.11)$ & $99.2 \%$ & $70.3 \%$ & $23.1 \%$ \\
\hline 16 & 8 & $20^{\circ}$ & $6.3(4.1)$ & $0.30(0.11)$ & $99.5 \%$ & $80.4 \%$ & $36.7 \%$ \\
\hline
\end{tabular}

$M$ - number of images in a subset, $\Delta$ - angle between consecutive images in a subset.

\section{Discussion}

The accuracy and robustness of registering a 3D preoperative image with 2D intraoperative images depends on the registration method and the anatomical structures that are to be registered. However, it is expected that the quality of $3 \mathrm{D} / 2 \mathrm{D}$ registration also depends on the number of $2 \mathrm{D}$ intraoperative images and the projections under which these images are acquired. In the past, researchers have used one, two or more intra-operative images but have not systematically studied the impact of X-ray views and their number on the performance of an intensity-based 3D/2D registration method [13], [14]. To confirm the expectations that better registrations results can be achieved with more intraoperative images and with images taken at certain projections, we have conducted a study using the recently proposed 3D/2D intensity-based registration method and images and "gold standard" registration data.

As expected, the accuracy of successful CT/X-ray registrations for different initial displacements increased when more X-ray images had been used. Using two instead of one X-ray image, more than doubles registration accuracy, i.e. from $0.9 \mathrm{~mm}$ to 0.4 $\mathrm{mm}$, which is more than enough for orthopedic procedures. The impact of the number of images used in registration accuracy could be explained with a fact that, when using a single X-ray image, the registration is well-defined for two in-plane translations and two in-plane rotations and ill-posed for one out-of-plane translation and one out-of-plane rotation $[9,13]$. By adding an additional X-ray image, with significantly different angle of view, one out-of-plane translation becomes in-plane and thereby well-defined. Our experimental results on vertebra data suggested that a 
significantly different angle of view between two images would be 20 degrees, where sufficient accuracy of $0.41 \mathrm{~mm}$ was achieved, while the best accuracy of $0.32 \mathrm{~mm}$ was obtained for almost perpendicular views (80 and 100 degrees). The minimal angle between views can be useful information for those clinical applications that cannot afford time and space for acquiring X-rays with perpendicular views. Regardless of the angle of view, one out-of-plane rotation still remains, even if more than two X-ray views are employed in a single plane, which is the case when using $\mathrm{C}$ arm with single rotation axis. However, even out-of-plane, the rotation could be welldefined, if the object of registration is not symmetric around one of its axis. This can explain our registration results, where adding additional X-ray images into the registration procedure has a small and clinically irrelevant impact on registration accuracy, since single vertebra body does not have symmetrical rotational axis. However, for other anatomy, for example for the shaft of the femur, which is close but not perfectly symmetric around one of the rotational axes, two X-ray images may not be sufficient to obtain the desired registration accuracy.

Similarly to registration accuracy, the percentage of successful registrations also increases where more X-ray images are used. The largest improvement is observed when deploying two, instead of one X-ray image, which can again be explained with ill-posed out-of-plane transformations. However, the percentage of successful registrations of $96 \%$ for initial displacement $0-6 \mathrm{~mm}(0 \div 17.2$ degrees), when using two X-ray views, would probably be a border line for most spine clinical applications. Our results suggested that the percentage of registration increases significantly when more than two X-ray views were used. Adding more X-ray images into registration process increases the statistical power of criterion function calculation. Moreover, the information from additional images can also reduce the effect of outliers, e.g. occlusions, which can be present on one of X-ray images but not on the other images, while outliers can impose additional local optima into criterion function. Both, the increase of statistical power and the decreased effect of outliers, result in a smoother criterion function, which reduces the probability of optimization algorithm to converge to some local optimum.

This study justified the assumption that more X-ray image views improve registration accuracy and reliability. For the given imaged anatomy, e.g. for single vertebra object, and the given intensity based registration method [2], at least two X-ray images should be employed in the registration process to achieve desire registration accuracy, while for desired registration reliability, more X-ray images should be used. On the other hand, increasing the number of X-ray images to increase the reliability of registration result in increases radiation and acquisition times. Some care has to be taken when generalizing our registration results to clinical applications. Even though a real cadaver anatomy was used in our experiments, our gold standard images lack the presence of soft tissue and ribs that can occlude vertebra on X-ray images and can consequently harm the registration. Moreover, the calibration of our X-ray imaging system is almost ideal in comparison with the calibration of standard $\mathrm{C}$-arm used in clinical procedures. By van Kraats et al. [13], it was reported that C-arm calibration errors have linear impact on registration errors. As a result, lower accuracy and reliability can be expected in real clinical use. However, we believe that our study provides general trends on how the number and the relative angle between X-ray image views affect the $3 \mathrm{D} / 2 \mathrm{D}$ registration and gives some useful guidelines for further studies. 
Acknowledgments. This work was supported by the Ministry of Higher Education, Science and Technology, Republic of Slovenia under Grant P2-0232.

\section{References}

1. Tomaževič, D., Likar, B., Pernuš, F.: Gold standard data for evaluation and comparison of 3D/2D registration methods. Comput. Aided Surg. 9, 137-144 (2004)

2. Tomaževič, D., Likar, B., Slivnik, T., Pernuš, F.: 3-D/2-D registration of CT and MR to Xray images. IEEE Trans. Med. Imaging 22, 1407-1416 (2003)

3. Galloway, R.L.: The process and development of image-guided procedures. Annu. Rev. Biomed. Eng. 3, 83-108 (2001)

4. Livyatan, H., Yaniv, Z., Joskowicz, L.: Gradient-based 2-D/3-D rigid registration of fluoroscopic X-ray to CT. IEEE Trans. Med. Imaging 22, 1395-1406 (2003)

5. Maurer, C.R., Fitzpatrick Jr., J.M., Wang, M.Y., Galloway, R.L., Jr., M.R.J., Allen, G.S.: Registration of head volume images using implantable fiducial markers. IEEE Trans. Med. Imaging 16, 447-462 (1997)

6. Colchester, A.C.F., Zhao, J., Holton-Tainter, K.S., Henri, C.J., Maitland, N., Roberts, P.T.H., Harris, C.G., Evans, R.J.: Development and preliminary evaluation of VISLAN, a surgical planning and guidance system using intra-operative video imaging. Medical Image Analysis 1, 73-90 (1996)

7. Grimson, W.E.L., Ettinger, G.J., White, S.J., Lozano-Perez, T., Wells III, W.M., Kikinis, R.: Automatic registration method for frameless stereotaxy, image guided surgery, and enhanced reality visualization. IEEE Transactions on Medical Imaging 15, 129-140 (1996)

8. Gueziec, A., Kazanzides, P., Williamson, B., Taylor, R.H.: Anatomy-based registration of CT-scan and intraoperative X-ray images for guiding a surgical robot. IEEE Trans Med Imaging 17, 715-728 (1998)

9. Weese, J., Penney, G.P., Desmedt, P., Buzug, T.M., Hill, D.L., Hawkes, D.J.: Voxel-based 2-D/3-D registration of fluoroscopy images and CT scans for image-guided surgery. IEEE Trans. Inf. Technol. Biomed. 1, 284-293 (1997)

10. Zheng, G.Y., Ballester, M.A.G., Styner, M., Nolte, L.P.: Reconstruction of patient-specific $3 \mathrm{D}$ bone surface from $2 \mathrm{D}$ calibrated fluoroscopic images and point distribution model. In: Larsen, R., Nielsen, M., Sporring, J. (eds.) MICCAI 2006. LNCS, vol. 4190, pp. 25-32. Springer, Heidelberg (2006)

11. Fleute, M., Lavallee, S.: Nonrigid 3-D/2-D registration of images using statistical models. In: Taylor, C., Colchester, A. (eds.) MICCAI 1999. LNCS, vol. 1679, pp. 138-147. Springer, Heidelberg (1999)

12. Fitzpatrick, J.M., West, J.B., Maurer Jr., C.R.: Predicting error in rigid-body point-based registration. IEEE Trans. Med. Imaging 17, 694-702 (1998)

13. van de Kraats, E.B., Penney, G.P., Tomaževič, D., van Walsum, T., Niessen, W.J.: Standardized evaluation methodology for 2-D-3-D registration. IEEE Trans. Med. Imaging 24, 1177-1189 (2005)

14. McLaughlin, R.A., Hipwell, J., Hawkes, D.J., Noble, J.A., Byrne, J.V., Cox, T.C.: A comparison of a similarity-based and a feature-based 2-D-3-D registration method for neurointerventional use. IEEE Transactions on Medical Imaging 24, 1058-1066 (2005) 